\title{
LA RELACIÓN DE DESARROLLO DE LAS ALIANZAS PÚBLICO PRIVADAS (APP) EN LA EDUCACIÓN SUPERIOR UN ENFOQUE INTEGRAL
}

\section{THE RELATIONSHIP OF DEVELOPMENT OF PUBLIC PRIVATE PARTNERSHIPS (PPPS) IN HIGHER EDUCATION A HOLISTIC APPROACH}

Claudia Preciado Awad claudia.preciado@urosario.edu.co Universidad del Rosario Bogotá- Colombia

Luisa Fernanda Garcia Lopez luisa.garcia@urosario.edu.co Universidad del Rosario Bogotá- Colombia

Fotografia de portada y Diagramación Sindy Catherine Charcas Ibarra 


\section{RESUMEN}

La investigación sobre la relación de desarrollo de las Alianzas público privadas (APP) en la educación Superior, estudio realizado de corte cualitativo de diseño descriptivo analítico, el cual inicia con la revisión de literatura en casos de países y universidades, permitiendo determinar cuáles son los casos más relevantes, el propósito de las APP confrontadas con sus problemáticas de instalación y ejecución, las fases empleadas en este estudio, parte de la elaboración de un estado del arte sobre el concepto, teorización de las APP y su marco legal, se analizó información de tres países que se fundamentan en el derecho anglosajón como lo son Estados Unidos, Australia y Reino Unido, el análisis y las discusiones se elaboraron desde el enfoque educativo constructivista, se caracterizó el caso desde la educación superior pública y privada en Colombia, relacionando las normas, su gobernanza y la estructura, los aportes más relevantes se dan en los desarrollos y problemáticas que se presentan en la aplicación de modelos de asociaciones público privadas , relacionando los factores que son causales de la falta de éxito de las APP como su difícil administración y sostenimiento, representados mediante convenios o figuras jurídicas utilizadas, su aporte permite la aproximación al fenómeno de la aplicación de las APP , investigación original centrado desde un enfoque de jurisprudencia e interpretación de desarrollo económico.

Palabras clave: derecho público, derecho administrativo, alianzas público privadas, concesión, Jointventure.

\section{ABSTRACT}

The research based on the relationship of development of public Private partnerships (PPP) in higher education, a study of qualitative of descriptive analytical design, which begins with the review of literature in cases of countries and universities; this helps to determine which are the most relevant cases, the purpose of the PPP confronted with their problems of installation and execution, the phases used in this study, part of the elaboration of a state of the art on the concept, theorization of the PPP and its legal framework, information from three countries were analyzed based on Anglo-Saxon law, such as the United States, Australia and the United Kingdom, the analysis and discussions were elaborated from the constructivist educational approach, the case was characterized from the Public and private higher education in Colombia, relating the norms, their governance and the structure, the most relevant contributions are given in the developments and problematics that are presented in the application of models of public private associations, relating the factors that cause the lack of success of PPP as their difficult administration and support, represented by conventions or legal etates used, their contribution allows the approach to the phenomenon of PPP, original research focused from an approach of jurisprudence and interpretation of economic development.

Keywords: public Law, administrative law, public-private partnerships, concession, Jointventure. 


\section{INTRODUCCIÓN}

La investigación propone una reflexión profunda sobre el concepto de las APP como ejercicio de construcción de modelos de desarrollo asociados a los propósitos de los países en vía de desarrollo, para la Mesa Directiva de la Conferencia Regional sobre Población y Desarrollo de América Latina y el Caribe CEPAL, $N$. en reunión extraordinaria realizada en Santiago en el mes de noviembre de 2017, como logro referido en este escenario se reconoce el amplio grado de trascendencia en la inversión extranjera directa para américa Latina, un aspecto que está en la relación del porque se incrementa estos dinamismos es la aplicación de la asociatividad y el desarrollo de propósitos comunes en la unión de los sectores más interesados, donde las alianzas público privadas cada día logran mayor comprensión en cómo se pueden implantar de manera eficiente y efectiva, en las asociaciones público-privadas (APP), se logra la alineación de los objetivos del sector público con los del contratista privado generalmente se logra utilizando incentivos informados por la teoría de la agencia mediante indicadores de gestión aplicados, relacionados para la toma de decisiones.

Sin embargo, el éxito del modelo de APP tradicional que ha sido cuestionado, y por sus acciones unilaterales, los nuevos procesos de APP se centran más en la colaboración y el intercambio de inversiones, para Solheim-Kile, E., \& Wald, A. (2019), se logra una ampliación de la visión transaccional cuando se alinean los propósitos y objetivos, el contratista generalmente utiliza incentivos informados por la teoría de la agencia o toma de decisiones mediante la elaboración de indicadores de gestión, se relaciona la teoría del capital social y la teoría de la autodeterminación que establece rutas para permitir una mejor comprensión de los incentivos para la alineación de objetivos al incluir aspectos motivacionales y relacionales, además de la visión transaccional, permitirá mediante los casos referidos y uso de casos múltiples que comprende cuatro proyectos de PPP de Noruega que sugieren varios beneficios de los mecanismos sociales y psicológicos para la alineación de objetivos en las PPP, incluidas la confianza, la reciprocidad y la motivación intrínseca.

La apuesta de las APP está relacionado con el papel las universidades que toma un valor importante en los mercados económicos debido a su impacto en los resultados de investigación, según Horner, S., Jayawarna, D., Giordano, B., \& Jones, O. (2019), sobre la elección estratégica en las universidades, como agencias de gestión y transferencia efectiva de tecnología, desarrollada, aplicado desde las alianzas con el sector productivo, para las alianzas público privadas el ingreso de conceptos técnicos como el apoyo a escala de la Oficina de Transferencia de Tecnología (TTO) y la provisión de incentivos, cada vez las universidades desde la investigación académica y las revisiones de políticas han resaltado la importancia potencial de las elecciones estratégicas hechas por los gerentes universitarios contribuyendo a la efectividad de la actividad de transferencia de tecnología, la teoría de la elección estratégica de Child como un marco integrador.

No obstante la puesta en práctica de un marco de elección estratégica y el uso de datos permitieron que el trabajo de Horner, S., Jayawarna, D., Giordano, B., \& Jones, O. (2019), aplicado a II5 universidades del Reino Unido reconoce que la infraestructura de la organización de soporte es necesaria pero no suficiente para explicar la eficacia de la transferencia de tecnología, el papel del empresa privada alienada con objetivos estatales como mediador estratégico, esta relación de mediación entre la alineación estratégica y la efectividad de la transferencia de tecnología está moderada por la amplitud de los esfuerzos de planificación estratégica, con aquellas universidades que involucran a un mayor número de profesores en los esfuerzos de planificación estratégica que se benefician más de la alineación entre las opciones estratégicas y la infraestructura de apoyo de la organización.

El estudio que se realizó por Horner, S., Jayawarna, D., Giordano, B., \& Jones, O. (2019), articula el papel de las Asociaciones Público-Privadas acorde a lo establecido en la ley colombiana como una posible herramienta para el desarrollo de la educación superior, teniendo en cuenta que esta figura jurídica ha sido pensada en Colombia para temas de Infraestructura principalmente vial, intentándose dar un primer paso según los estudios realizados por el Departamento de Planeación Nacional en materia de construcción de edificaciones para la prestación de servicios de Educación primaria y secundaria, pero no siendo así en materia de educación superior. No obstante, lo anterior, en Colombia existe varios ejemplos de alianzas o asociaciones publicas privadas que han contribuido en el desarrollo la educación superior que sirven como base para nuevas iniciativas importantes para el país.

\section{CONCEPTUALIZACIÓN DE LAS ALIANZAS PÚBLICO-PRIVADAS}

Un acercamiento al concepto de Alianzas o Asociaciones Público- Privadas (APP) han sido definidas de diversas maneras, una de ellas la establece Al-Said Albella, A. (20I2), las alianzas público-privadas son la relación de los bienes servicios y alianzas transformacionales, para resolver una problemática, otro acercamiento el de Growe, D. (20I0), aplicado según la United Nations Foundation (I73); se conceptualizan como las APP como la confluencia de actores, estatales, privados, sociedad civil, que tiene como propósito una agenda compartida de interés común y público y que implica que se hace de manera voluntaria en cada involucrado, se comprometen a la optimización de los recursos (compartir), relacionando mediante acuerdos lo más explícitos posibles con propósitos claros y objetivos, compartiendo riesgos y estímulos, estas acciones encaminadas en un marco de construcción de confianza y legitimidad, permitiendo obtener beneficios en común, donde no se delega responsabilidades.

Las alianzas público privadas sirven de base en los planes de desarrollo de los países, organizan para propósitos comunes, para Paletta, A., Fava, F., Ubertini, F., Bastioli, C., Gregori, G., La Camera, F., \& Douvan, A. R. (2019), las APP cumplen con su función de asociatividad entre las Universidades, las industrias y desarrollo sostenible, logrando respuestas fortalecidas por estos estamentos, ejemplo de aplicación se da en el Ministerio de Medio Ambiente del G7 de 2017, bajo la Presidencia italiana que tuvo lugar en Bolonia, Italia, del I I al I 2 de junio de 2017, donde los representantes de Universidades e 
Institutos de Investigación y de las principales industrias de los países del G7 se unieron para identificar las prioridades actuales y futuras para la implementación del Desarrollo Sostenible en los países del G7, el papel de las universidades con un enfoque en la enseñanza y el aprendizaje sostenible, la investigación universitaria y la cooperación efectiva entre universidades e industrias, estos resultados inherentes en la co construcción de políticas públicas desde sus interacciones, las universidades tienen la responsabilidad central de promover un enfoque sistémico en relación con los múltiples desafíos sociales.

No obstante, el Británico Jhon Davie, presidente de Altra Capital Limited, en su libro de mano del año 20I3, “ some helpful knowledge and experience that can make the difference between success and failure of PPP program" define a las APP como un acuerdo contractual entre una entidad gubernamental y una empresa privada que incentiva a la empresa privada a diseñar, construir, mantener y a infraestructura pública financiera de manera eficaz y eficiente", Davis, N, \& Luna, P. M. (20I3), siendo esta una definición más concreta de los que se desea principalmente con una APP como lo es el diseño, construcción mantenimiento y financiación de infraestructura incluyendo dos principios básicos para la realización de las mismas que son los de efectividad y eficiencia.

\section{POSTURAS TEÓRICAS APLICADAS EN LAS ALIANZAS PÚBLICO PRIVADAS APP}

Para este análisis se tiene una relación directa con las teorías de la inclusión social y del capital social de Pierre Bourdieu (1997), la teoría de la autodeterminación (Ryan, Kuhl, \& Deci, 1997)., la teoría de la elección estratégica de Child (1972 y 1997) como un marco integrador para entender cómo se aplican las APP, la teoría de la modernización Neil J. Smelser (1995) que dialogan indirectamente con las alianzas públicos privadas existentes.

Las APP para comprensión o definición trabajan el concepto de la teoría de la modernización, Parsons, T., \& Neil, J. (1984). Smelser, 1956, quien refiere su teoría al cómo se integra socialmente la economía a los sectores públicos y privados, las APP ayudan a la mejor comprensión de las sociedades modernas donde logran ser más productivas, si se vinculan a la población, cada uno de sus componentes integrados, la educación para los infantes es de mayor calidad, la población menos favorecida recibe su atención aplicada para lograr un avance, el análisis de Smelser, afirma que las sociedades modernas tienen el aspecto individual de la diferenciación estructural particular, es decir, una definición clara de las funciones y papeles políticos de las instituciones, que aunque la diferenciación estructural ha aumentado la capacidad funcional de las instituciones modernas, refiere de una asociación con sectores no vinculados, como las universidades y el estado, a la vez también ha creado un problema de integración y de coordinación de las actividades de diferentes nuevas instituciones, las cuales tienen un propósito que conlleva al desarrollo, una de las derivaciones de estos planteamientos, de manera concreta, es el concepto de los pesos y contrapesos que se tienen en los sistemas institucionales en países más desarrollados, los poderes públicos están más limitados en sus funciones y existe cierto control no sólo por parte del electorado, sino también de otras instancias de la institucionalidad de las naciones., en América Latina uno de los problemas de las democracias ocurre en función de que no existe la fortaleza institucional de estos pesos y contrapesos, los partidos políticos no son por lo general instituciones de intermediación social y no hay mecanismos de revocabilidad, estos argumentos son los principales detractores de la política de aplicación de las APP, referente de la teoría de la modernización.

La teoría de la inclusión social y del capital social de Pierre Bourdieu, relaciona la aplicación de las alianzas público privadas como el factor diferencial de acuerdos entre las dos partes, debido a que desde la teoría el capital social según Bourdieu, P. (1997), parte el capital cultural inherente en la sociedad, su relación con la escuela y como este genera un espacio social determinado para el desarrollo, las alianzas y acuerdos por el desarrollo, que no son totalmente independientes del capital económico y cultural de un individuo determinado, ni del de la totalidad de individuos relacionados en estos acuerdos, si bien no es menos cierto que no puede reducirse la responsabilidad que tienen los involucrados en las APP, donde se comparte la misionalidad y riesgos de este propósito de alianza, aspecto generado por las relaciones de intercambio presupone el reconocimiento de un mínimo de homogeneidad objetiva entre quienes mantienen dichas relaciones, así como al hecho de que el capital social ejerce un efecto multiplicador sobre el capital efectivamente disponible logrando que este factor juegue a favor de la población que requiera de una asociación de voluntades, por esto es crucial determinarlo en las alianzas público privadas existentes.

La teoría de la autodeterminación (Ryan, Kuhl, \& Deci, 1997). Constituye un aporte significativo al entendimiento del éxito o el fracaso de los acuerdos en los sectores públicos y privados, según Ryan, R., \& Deci, E. L. (2000), se constituye en la facilitación de la motivación intrínseca, permitiendo el desarrollo social, y el bienestar común, los seres humanos pueden ser proactivos y comprometidos o, alternativamente, pasivos y alienados, en gran medida como una función de las condiciones sociales en las cuales ellos se desarrollan y funcionan, la teoría de la autodeterminación se ha focalizado sobre las condiciones del contexto social que facilitan versus las que previenen los procesos naturales de la auto-motivación y el desarrollo psicológico. examinando los factores que amplían versus que reducen la motivación intrínseca, la autorregulación. Se postulan sobre las tres necesidades psicológicas innatas competencia, autonomía, y relacionarse las cuales cuando son satisfechas producen la ampliación de la auto motivación y la salud mental y cuando son frustradas llevan a la reducción de la motivación se convierte en un enfoque hacia la motivación humana y la personalidad que usa métodos empíricos tradicionales mientras emplea una meta teoría organísmica que enfatiza la importancia de la evolución de los recursos humanos internos para el desarrollo de la personalidad y la autorregulación de la conducta (Ryan, Kuhl, \& Deci, 1997).

Para la teoría de la autodeterminación se identifican los factores ambientales que estorban o reducen la auto motivación, el funcionamiento social, y el bienestar personal, donde se delega y se reciben los acuerdos con las responsabilidades, el entendimiento de esta teoría permite encontrar las variables 
de retrocesos, aunque muchos efectos perniciosos han sido explorados, es concerniente no sólo a la naturaleza específica de las tendencias positivas del desarrollo, sino que también examina los ambientes sociales que son antagonistas a esas tendencias, el continuo de la Autodeterminación que muestra los tipos de motivación con sus estilos regulatorios, el locus de causalidad y los procesos correspondientes presentes en las alianzas público privadas se describen en la tabla 0I, allí se establecen los ciclos presentes en el éxito o el fracaso de una APP.

Tabla 0I.

Tipos de motivación regulatorios presentes en las alianzas público privadas.

\begin{tabular}{|c|c|c|c|}
\hline Conducta & No. autodeterminada & \multicolumn{2}{|c|}{ Autodeterminada } \\
\hline Motivación & Desmotivada & Motivación Extrínseca & Motivación Intrínseca \\
\hline Estilos regulatorios & No-regulación & $\begin{array}{l}\text { Regulación Externa Regulación } \\
\text { Introyectada Regulación } \\
\text { Identificada Regulación } \\
\text { Integrada }\end{array}$ & Intrínseca \\
\hline $\begin{array}{l}\text { Locus de casualidad } \\
\text { percibio }\end{array}$ & Impersonal & $\begin{array}{c}\text { Externo Algo Externo Algo } \\
\text { Interno }\end{array}$ & Interno \\
\hline $\begin{array}{l}\text { Proceso regulatorios } \\
\text { relevantes }\end{array}$ & $\begin{array}{l}\text { No-intencional No-evaluativo } \\
\text { Incompetencia Falta de control }\end{array}$ & $\begin{array}{l}\text { Obediencia Recompensas in- } \\
\text { competencia Falta de control; } \\
\text { Castigos Internas y Consciente } \\
\text { el YO Externas y Auto-control } \\
\text { Ego-implicación Recompen- } \\
\text { sas Importancia Personal Va- } \\
\text { lor Congruencia Síntesis con } \\
\text { Consciencia }\end{array}$ & Interés gozo satisfacción \\
\hline
\end{tabular}

Fuente: Ryan, R., \& Deci, E. L. (2000). La Teoría de la Autodeterminación y la Facilitación de la Motivación Intrínseca, el Desarrollo Social, y el Bienestar. American Psychologist, 55(I), 68-78.

La teoría de la elección estratégica de Child ( 1972 y 1997), se ubica en este aporte desde el conocimiento integral de la organización, que mediante alianzas, acuerdos y espacios voluntarios integran el conocimiento a su sector más práctico y especifico, no obstante, se refiere las APP como el elemento integrador de esta teoría que desde los aportes mencionados de Child se observa como un proceso cognitivo que se lleva a las organizaciones, entonces las empresas de índoles diferentes a los públicos o privadas, pueden generar opciones de selección, como consecuencia, los valores pueden legitimar o sancionar alternativas estratégicas como deseables, aceptables, esta condición le permiten a las APP aplicar en las escalas de medición de los factores de la elección estratégica, aspectos directos de los acuerdos voluntarios que proponen desde problemáticas irresolubles el aporte y el concierto de todos los actores de la región, para Zapata Rotundo, G. J., Canet Giner, M. T., \& Peris Bonet, F. J. (2008), ya es un aspecto de la Visión directiva y elección estratégica, como las dinámicas organizaciones llevan a nuevas maneras de influenciar en los tipos básicos de trabajo, el entorno de la organización: un

\section{Conceptos relevantes de las APP}

Para ahondar en los términos relacionados en la investigación se debe conceptualizar aspecto y términos concretos desde los marcos jurídicos mundiales, para ello se debe acercar a una definición particular como las Figuras Jurídicas; las Asociaciones Público- privadas se han venido estudio de sus tipologías y su vinculación con la percepción directiva y el diseño organizativo, sin embargo, se requiere de una lectura integral de las responsabilidades para determinar la elección estratégica adecuada para la implementación de las APP.

Esta elección estratégica como las teorías mencionadas logran un acercamiento a las definiciones referenciadas, se determina que las Alianzas Publico Privadas son una forma de relacionamiento entre entidades públicas y privadas en la búsqueda de mejores condiciones para una comunidad principalmente en el área de los servicios o bienes públicos teniendo como pilares la eficiencia y efectividad tanto en los recursos como del proyecto planteado, respetando los aspectos de selección y escogencia del recurso humano, logrando fortalecer la autodeterminación en cada reto propuesto, para las APP su aplicación y exitoso proceder logra un desarrollo cuando se clarifica estas aplicaciones teórico conceptuales .

desarrollando a través de diversas figuras jurídicas, como lo son la concesión, el Jointventure, los Convenios de asociación entre otros siendo estos tres los más comunes.

La Concesión, este concepto refiere a el contrato que 
ha sido la figura jurídica más utilizada en la conformación de las APP, principalmente en lo relacionado con obras públicas, el cual se ha considerado de naturaleza jurídica mixta "ya que dicho acuerdo de voluntades participa del régimen jurídico del contrato de obra y de la concesión de servicios públicos.”(Urueta, J., 20I0).

Para Sepúlveda Figueroa, B. J. (2014), a partir de esta normatividad se han desarrollado cuatro tipos de generación de concesión donde en la última de ellas contó con sistemas más sofisticados de distribución y asignación de riesgos, pero los diseños del proyecto estarían a cargo de un tercero, en la Concesión de obras públicas se pueden identificar claramente dos etapas: una, relacionada con la construcción de la obra; otra referida a la explotación económica de la misma(Urueta Rojas, 2010, p.32).

La figura jurídica denominada Jointventure, se utiliza en la celebración de Asociaciones Público - Privadas ya que como figura jurídica es utilizada para un negocio especifico, uniendo lo mejor que tiene cada una de las partes que conforman el mismo, en el contrato de "joint venture" es un negocio jurídico complejo que tiene por objeto el establecimiento de una unión de esfuerzos, a través de aportes de diversa índole, que pueden consistir en transferencias de activos, materia prima, capital, tecnología, know-how; ventas, canales de distribución, personal, financiamiento o productos, en el caso de los "joint ventures" con participación pública también es frecuente el aporte de pólizas de garantías o subsidios pertinentes, en este tipo de asociación no existe animus societatis por que las partes suelen seguir operando sus actividades de manera independiente y la unión empresarial ocurre puntualmente frente a un negocio en particular" (Urueta Rojas 2010), la idea base de formar un joint venture es compartir conocimientos similares, aptitudes y recursos, compartiendo a su vez las ganancias y los riesgos, es una asociación en donde las partes que en este caso será el Estado y el particular, tienen fines y obligaciones en común.(Erazo Chapid 2017).

Los convenios de asociación, para la Constitución Política de Colombia de 1991 en su art. 355 establece. “. El Gobierno, en los niveles nacional, departamental, distrital y municipal podrá, con recursos de los respectivos presupuestos, celebrar contratos con entidades privadas sin ánimo de lucro y de reconocida idoneidad con el fin de impulsar programas y actividades de interés público acordes con el Plan Nacional y los planes seccionales de Desarrollo. El Gobierno Nacional reglamentará la materia." Como parte de la reglamentación que surge a partir de este artículo de la Constitución Nacional se expide la ley 489 de 1998 el cual en su art. 96 señala: "Constitución de asociaciones y fundaciones para el cumplimiento de las actividades propias de las entidades públicas con participación de particulares. Las entidades estatales, cualquiera sea su naturaleza y orden administrativo podrán, con la observación de los principios señalados en el artículo 209 de la Constitución, asociarse con personas jurídicas particulares, mediante la celebración de convenios de asociación o la creación de personas jurídicas, para el desarrollo conjunto de actividades en relación con los cometidos y funciones que les asigna a aquéllas la ley. Los convenios de asociación a que se refiere el presente artículo se celebrarán de conformidad con lo dispuesto en el artículo 355 de la Constitución Política de Colombia, en ellos se determinará con precisión su objeto, término, obligaciones de las partes, aportes, coordinación y todos aquellos aspectos que se consideren pertinentes..."

Definición de instituciones públicas en Colombia, las clasificaciones de la educación superior en Colombia se dan de acuerdo a su naturaleza jurídica, por lo cual para la investigación se dio el análisis en lo referente a las instituciones públicas, encontrándose en el art. 57 de la ley 30 de 1992 que: "Las universidades estatales u oficiales deben organizarse como entes universitarios autónomos, con régimen especial y vinculados al Ministerio de Educación Nacional en lo que se refiere a las políticas y la planeación del sector educativo". Igualmente, el artículo 58 establece que "La creación de universidades estatales u oficiales y demás instituciones de Educación Superior corresponde al Congreso Nacional, a las Asambleas Departamentales, a los Concejos Distritales o a los Concejos Municipales, o a las entidades territoriales que se creen,".

Las Instituciones Públicas ya sean de orden nacional, departamental o municipal cuenta con aportes del presupuesto nacional colombiano y de las entidades territoriales y como complemento de lo anterior, el gobierno creó una Estampilla Pro Universidad Nacional, cuyos recursos son recaudados y administrados por el Fondo Nacional de Universidades Estatales creado mediante el art 10 de la ley 1697 de 2013, el cual también se encarga de manejar los recursos de las estampillas de las demás universidades estatales.

\section{Metodología}

Para la investigación realizada sobre la relación de desarrollo de las Alianzas público privadas (APP) en la educación Superior, se remonta a un estudio realizado de corte cualitativo de diseño descriptivo analítico, según Hernández Sampieri, R., Fernández Collado, C., \& Baptista Lucio, P. (20l0), con una metodología de investigación cualitativa, parte de la revisión de literatura desde un enfoque social que fundamente el propósito de las APP, en un estudios de Aguja, F. A. P., \& Quintero, H. J. G. (2017), la metodología de enfoque social se puede considerar como la ruta de la innovación social encaminada a la aplicación de actores estatales que pueden considerar la aplicación de enfoques legales y de aproximaciones teóricas, los resultados se presentan en todo el proceso de construcción del documento, esta reflexión se realiza con aportes desde la innovación social en la cual se aplicó las APP, las fases empleadas en este estudio entregan un estado del arte sobre el concepto, la teorización y el marco legal que desarrolla el estudio, los antecedentes se hacen de casos aplicados en países en desarrollo, se analizan los modelos de APP en Educación Superior que se vienen desarrollando en el ámbito internacional tomando como referencia (3 ) países de derecho anglosajón como lo son Estados Unidos, Australia y Reino Unido, el análisis se plantea mediante la relación de conceptos, se generaron matrices de revisión de literatura, aplicado de manera cronológica asociado por categorías de análisis, los antecedentes de aplicación jurídica, discusiones desde el enfoque educativo, la relación permite que mediante un enfoque hermenéutico 
crítico asociados a la teorías presentadas en el marco teórico, se analizó la completitud de las normas, el caso específico lo establecen instituciones comparadas desde la ubicación de la Institución de educación superior en la ciudad de Bogotá.

Se trabajaron con 2 universidades Nacionales Publicas y 2 Universidades Nacionales Privadas, 3 análisis de países

\section{Resultados}

Tabla 02

Estado del Arte Antecedentes 3 grandes regiones internacionales donde se aplica APP, permitiendo determinar cuáles son los casos más relevantes, el propósito de las APP confrontadas con sus problemáticas de instalación y ejecución, las fases empleadas en este estudio, parte de la elaboración de un estado del arte sobre el concepto, teorización de las APP y su marco legal, se analizó información de tres países.
Antecedentes históricos, regiones UK, Europa y USA

El Derecho comparado se inicia desde los aportes de los antecedentes históricos, en las regiones UK, Europa y USA; donde las Asociaciones Publico Privadas o las PPP's por sus siglas en inglés (Public Private Partnership) inician en Gran Bretaña, en el gobierno conservador de John Major bajo el nombre de PFI (private Finance Iniciative) el cual fue "un programa diseñado para la efectiva inversión privada en las obras públicas, cuyo objetivo era el de disminuir el riesgo de las obras y las infraestructuras en cabeza del Estado, este programa en 1992 tuvo un apoyo mayor con el gobierno de Tony Blair, elegido en 1997, quien amplió la iniciativa $\mathrm{PFI}$, pero trató de cambiar el énfasis a la realización de "valor por dinero",principalmente a través de una asignación adecuada de los riesgos".

las Antecedentes históricos región india, Bangladesh

Antecedentes Latina

A partir del año 2005 el gobierno de la India ha venido implementando diferentes mecanismos para la evaluación de las APP'S, es así como la Comisión de Asuntos Económicos del Consejo de Ministros aprobó el procedimiento para la aprobación de proyectos de la Asociación del Sector Público Privado (PPP) del Sector Central. En la actualidad el departamento de Asuntos económicos del Gobierno de la India cuenta con una página denominada public private parnetship in india www.pppinindia.gov. in, en donde se pueden consultar toda las circulares que al respecto se han emitido, los modelos para desarrollar las APP'S y una base en donde se puede observar cuantos y que tipos de proyectos se han recomendado, encontrándose que del 20 de diciembre de 2005 al 17 de mayo de 2 Del 20 de diciembre de 2005 al 17 de mayo de 2017 lo han sido 3 I I proyectos, básicamente en proyectos de carreteras, vías y aeropuertos.
En México se expide el 16 de enero de 2012 la ley de Asociaciones públicos privadas, las cual se rige por los principios de los art. 25 y 134 de la Constitución política de los Estados Unidos Mexicanos tal y como lo indica su artículo primero, Hernández, D. M. M., \& Díaz, L. F. (20l8), los cuales hacen referencia al desarrollo económico nacional y a sus recursos económicos, estableciéndose en su art. 3. “También podrán ser proyectos de asociación público -privada los que se realicen en los términos de esta ley, con cualquier esquema de asociación para desarrollar proyectos de inversión productiva, investigación aplicada y/o de innovación tecnológica.", con lo cual no solo se desarrolla la parte de infraestructura de dicho país, si no que se le da una gran relevancia a la investigación y la innovación tecnológica. Esta ley fue reformada parcialmente, el 21 de abril de 2016. En este país igualmente existe un Centro para el Desarrollo de Infraestructura y Servicios en Asociación Público-Privada el cual surge en el marco del Convenio de Colaboración (2010) entre el Tecnológico de Monterrey y el Fondo Multilateral de Inversiones del Banco Interamericano de Desarrollo (FOMIN/BID), programa Fortalecimiento de Municipios para el Desarrollo de Proyectos de Asociación Público-Privada (MuniAPP). El objetivo del programa es promover la expansión y mejora de servicios y obras públicas a través de inversiones en la modalidad de Asociación Público-Privada (APP). ("Centro para el Desarrollo de Infraestructura y Servicios en Asociación Público-Privada» s. f.). 
Según Erazo Chapid, E. (2017), las PFI Ac posteriormente conocidas como PPP se viene utilizando en Gran Bretaña desde la $\&$ década de los 90 's para realizar diferentes proyectos en áreas como la salud, la educación y militar, la cual empezó a tener una caída en número de proyectos a partir del año 2008. Las PPP's solo far se desarrollaron en Gran Bretaña, si no que se expandió rápidamente por gran parte de Europa en países como Francia Alemania y España. así como por USA, Canadá y Australia.

En Francia se comenzó a usar el término Partenariat y en el año de 2004 se expide la ley sobre los contratos de Partenariat definiendo este como un contrato administrativo en donde el Estado $\circ$ un Establecimiento público del Estado confían a un tercero durante un tiempo determinado el mantenimiento, la explotación de obras de un servicio público (Ordonnance $n^{\circ} 2004-559$ du 17 juin 2004 sur les contrats de partenariat. s. f.). Esta ley es derogada en el año 2015, Vandepoorter, A. (2014), siendo tratado todo lo referente al Partenariat en la ley relativa a los Mercados Públicos en donde se establece como un contrato público que permite a un operador económico $\circ$ un grupo de operadores económicos encargarse de una tarea global (Ordonnance $n^{\circ} 2015-899$ du 23 juillet 2015 relative aux marchés publics s. f.).

Así mismo en España en el año 2007 se introduce el contrato típico de la colaboración público-privada a través de la ley de Contratos del Sector Publico en su art. II, define este de manera muy similar al contrato de Partenariat de Francia, mencionado anteriormente, Iglesias, J. L. V. (2008). Comentarios a la Ley 30/2007, de 30 de octubre, de contratos del sector público. La Ley. Las PPP se han utilizado en los Estados Unidos en una amplia gama de sectores en varias formas durante más de 30 años. Sin embargo, los presupuestos públicos cada vez más acentuados y un impulso sostenido hacia el alivio fiscal llevado a cabo en todos los niveles de gobierno han hecho que el uso de las PPP sea más común. (Robert $\mathrm{H}$. Edwards Jr., apr2015), pero es el año 2014 en la administración de Obama donde se le da un mayor impulso con un nuevo enfoque.
Adams, A. M., Rabbani, A., Ahmed, S., Otro de los países de América latina que Mahmood, S. S., Al-Sabir, A., Rashid, S. F., cuenta desde el 2008 con una Ley Marco \& Evans, T. G. (20I3), otro de los países de Asia con un importante desarrollo de APP'S es Bangladesh, quien, en agosto de 2010, emitió la Política y Estrategia para la Asociación Público-Privada (PPP) para facilitar el desarrollo de la infraestructura y los servicios públicos básicos del sector vital para la población de Bangladesh. El programa PPP forma parte de la meta del Gobierno de Visión 202I de asegurar una trayectoria de crecimiento más rápida e inclusiva y de satisfacer mejor la necesidad de servicios públicos mejorados y de alta calidad de una manera fiscalmente sostenible. («Public Private Partnership Authority Bangladesh» s. f.)

En Bangladesh se crea una oficina independiente que depende del primer ministro para apoyar los proyectos de APP. En la actualidad los principales sectores donde se desarrollan proyectos son los de Salud, Transporte, infraestructura social, educación, energía, turismo, alojamiento civil, textil e investigación y desarrollo. de Asociaciones Público - Privadas es Perú, mediante el Decreto legislativo 1012 del 13 de mayo de 2008, siendo el punto de partida para la expedición de diferentes leyes y decretos que reglamentan y promueven este tipo de Asociaciones, promulgándose en el año de 2015 el Decreto Legislativo del Marco de Promoción de la Inversión Privada mediante Asociaciones Público - privadas. Estrada, V. (2017) donde se resalta como se ha realizado esta implementación en Latinoamérica, de igual forma cuenta con una Agencia de Promoción de la Inversión Privada - PROINVERSIÓN, el cual "es un organismo público ejecutor, adscrito al Ministerio de Economía y Finanzas, con personería jurídica de derecho público, autonomía técnica, funcional, administrativa, económica y financiera. Constituye un pliego presupuestal.”(«Prolnversión :: Proinversión»s. f.) 
La tabla 02, relaciona el enfoque de cada evento realizado en las 3 grandes regiones, allí se plasma el avance e importancia de las alianzas público privadas como un instrumento de conocimiento para el desarrollo, algunos casos encontrados visibilizan que no es fácil la consecución de estos acuerdos, allí se relaciona el papel de la autodeterminación como la teoría que facilita el bienestar de cada país quienes buscan soluciones en su población, pero lo rescatable es el fortalecimiento del capital social, articulado desde la elección directiva y voluntad política de cada estamento involucrado, la ciencia, la tecnología y el desarrollo mundial se articula con las 3 grandes regiones US, Unión europea y Latinoamérica, se rescata de estos resultados su contribución y características.

\section{Estado del arte de los inicios de las APP en Colombia (caso de estudio)}

En Colombia, con la Constitución Política del año de 1991, se introducen nuevos conceptos e importantes cambios, imponiéndose al Congreso de la República por ejemplo en el último inciso de su artículo I50, la competencia de expedir estatuto general de contratación de la administración pública y en especial de la administración nacional, norma que se fue expedida dos años más tarde mediante la ley 80 de 1993, donde se regulan los contratos del Estado y su relación con los particulares, la cual fue modificada por la ley I I 50 de 2007.

Debido a esta nueva normativa es a partir de los años noventa que se empiezan a realizar varias asociaciones entre las entidades públicas y las privadas para la construcción de diferentes obras, con en el caso de la ciudad de Bogotá donde se realiza la construcción de la Biblioteca Pública y Centro Cultural Julio Mario Santo Domingo, la cual se lleva a cabo por una alianza entre la Fundación Julio Mario Santo Domingo y la Secretaría de Cultura, Recreación y Deporte Distrital. Según Vásquez Jaramillo, F. (2017), en el estudio realizado rescata las fallas y retos de los modelos APP desde la consolidación social. Es este tipo de asociaciones las que llevan a pensar al gobierno distrital en el año 201 I la creación de un Manual de Asociaciones Público-privadas, así como la expedición de un decreto sobre política pública de cooperación, en donde las APP tenían un papel muy importante y estableciéndose en el art. 9 Numeral 3 “ Las alianzas público-privadas, corresponsabilidad y responsabilidad social: Las Alianzas público-privadas, constituyen un escenario para que el Distrito Capital y el sector privado, incluyendo la sociedad civil, se asocien para desarrollar acciones coordinadas de mutuo beneficio, generando condiciones de confianza que tengan alto impacto en la sociedad" , (Planeación, 20 I I). Finalmente, este decreto no es expedido por las Administración Distrital, a pesar de los estudios y esfuerzos realizados.

Sin embargo, en el año 2012 la ley 1508 se convierte en la primera en regular el régimen jurídico de las asociaciones público privadas en Colombia, estableciendo en su artículo primero que" Las Asociaciones Público Privadas son un instrumento de vinculación de capital privado, que se materializan en un contrato entre una entidad estatal y una persona natural o jurídica de derecho privado, para la provisión de bienes públicos y de sus servicios relacionados, que involucra la retención y transferencia de riesgos entre las partes y mecanismos de pago, relacionados con la disponibilidad y el nivel de servicio de la infraestructura y/o servicio.". Con el fin de reglamentar la ley 1508 de 2012, según Sepúlveda, B., \& Blanca, J. (2014), en Colombia se han expedido diversos decretos en el transcurso de 5 años, tales como D. 1467 de 20I2, Decreto 100 de 20I3, D. I6I0 de 20I3, D. 301 de 20I4, D.I553 de 2014, D. 2043 de 2014, todos estos compilados en el Decreto 1082 de 2015 Libro 2 Capitulo 2 Titulo 2, generándose en los 3 primeros años un clima de inseguridad jurídica y de desconfianza por parte del sector privado a raíz de este cambio de normatividad en tan poco tiempo esto se resalta porque es una variable que toda APP enfrente que es el marco legal Legislativo, O. (20/2). Asociaciones Público Privadas Ley 1508 de 2012. Boletín, 197.

Según los autores Erasso Cifuentes, M. C., Pérez Soto, M., Pimienta Tabera, J. G., Rendón Ossa, E., \& Vásquez Zapata, C. D. (20|4), se debe reconocer todos los cambios jurídicos conceptuales con los que se conforman las APP donde se puede establecer la trazabilidad y formas de actuación, las APP en Colombia se pueden clasificar de acuerdo con la normatividad referenciada en de iniciativa privada e iniciativa pública, así como por los sectores en que funciones estas, un primer sector es en "infraestructura productiva. Conformada por todas aquellas obras físicas que permiten elevar los niveles de producción y eficiencia de los sectores que componen la oferta productiva de un país y que contribuyen al crecimiento de la economía. Ejemplos de proyectos de este tipo se encuentran en el transporte, la movilidad urbana, las comunicaciones y la logística.", para el Departamento Nacional de Planeación de Colombia (DNP) (20l3), el otro sector es el de "En infraestructura social. Está conformada por las obras y servicios relacionados que permiten incrementar el capital social de una comunidad y su posibilidad de acceder a mayores servicios y/o de mejor calidad. Hacen parte de la infraestructura social sectores y proyectos en Educación: colegios para preescolar, básica y media; establecimientos e institutos de educación superior. Salud: hospitales, centros de salud primaria. Edificaciones públicas: edificación de oficinas públicas y de la rama judicial. Deporte y cultura: escenarios deportivos, artísticos y culturales. Defensa y penitenciaria: cárceles, centros de detención preventiva. (Departamento Nacional de Planeación (DNP), s.f.)

\section{Modelos de asociaciones público privadas en la educación superior estado del arte (casos de estudio)}

El Banco Mundial ha sido desde hace unos años uno de los principales promotores de las Asociaciones Publico Privadas en el área Educativa, siendo estas consideradas dentro de sus estrategias para el desarrollo de la misma, tal y como lo señalan Verger, A., \& Bonal, X. (20I2), se debe incluir en la agenda global el programa de las PPP, este también 
ha penetrado considerablemente en la última estrategia del sector educativo del Banco Mundial (conocida como Estrategia 2020). Dicha estrategia, que aspira a establecer las prioridades de la reforma educativa en los países en vías de desarrollo en la década actual, considera que para mejorar los resultados de aprendizaje en la mayoría de países hay que centrarse en reformar la 'gobernanza' de los sistemas educativos. Específicamente, la estrategia propone una restructuración educativa profunda mediante la reintroducción de mecanismos de financiación competitiva como los vouchers' y la elección de centro educativo $y$, en general, otorgar mayor importancia al rol que puede jugar el sector privado.

En esta estrategia, por primera vez, el Banco Mundial hace explícita la creencia de que la implicación del sector privado en la educación puede beneficiar directamente a los pobres. Aunque se supone que el sector privado es principalmente para los estudiantes que pueden permitirse pagar por sus servicios, las instituciones privadas son importantes proveedores de servicios educativos para incluso las comunidades más pobres, especialmente en áreas en las que los gobiernos no pueden llegar (World Bank 20II, p. 20). Es por lo anterior se analizaron los modelos de APP en Educación Superior que se vienen desarrollando en el ámbito internacional tomando como referencia (3) países de derecho anglosajón como lo son Estados Unidos, Australia y Reino Unido, siendo este último como ya se mencionó el país donde surgen todo este movimiento de las APP; para entrar a analizar posteriormente los algunos casos en Colombia de Alianzas Públicos Privadas en el área de la Educación Superior.

Tabla 03.

Análisis de 3 regiones APP, orígenes e casos de análisis

\section{USA}

Estados Unidos es uno de los referentes más cercanos para Colombia, el cual lleva varios años desarrollando la figura de la APP's y en donde si bien no existe una legislación del orden nacional, $s$ existen diversas leyes acordes a cada estado federado, los requisitos para los contratos de APP tienen su origen en la legislación habilitante, proporciona la autoridad legal para que la agencia estatal ingrese en una APP, los términos y condiciones del contrato de APP deben encajar perfectamente dentro del alcance de la legislación habilitante Identifica la implementación de agencia y describe los tipos de proyectos permitidos bajo las estructuras APP, también puede identificar las fuentes de financiamiento público y otros requisitos para que un contrato de APP cumpla con la legislación habilitante.

Las Universidades de Estados Unidos tanto públicas con privadas han venido utilizando las APP como una herramienta para mejorar su infraestructura y los servicios para la comunidad estudiantil, es así como en el Estado de Kentucky en septiembre de 2013, la Universidad de Kentucky emitió una solicitud de propuestas que buscan un operador privado para llevar potencialmente el comedor del campus operación de los servicios en un esfuerzo por ampliar la capacidad de las instalaciones de comedor en medio de una mayor esfuerzo de

Australia

UK

El Reino Unido

En Australia las APP's en Educación Superior se han venido utilizando más para el fortalecimiento de la educación y la investigación que para la infraestructura de las Instituciones Universitarias, es así como podemos encontrar los casos de la Universidad Central de Queensland como uno de los referentes iniciales de estos tipos de iniciativas en este país, o el de los Centros Cooperativos de Investigación (CRC) por sus siglas en ingles que aún se encuentra en desarrollo. Estos dos casos serán revisados a continuación:

a. La Universidad Central de Queensland: en los años noventa junto con un empresario privado (Skinner) crean la asociación CQU-CMS con el fin de desarrollar más la educación a distancia con un enfoque principal en los estudiantes extranjeros, convirtiéndose esta alianza según Paul Rodan en: "un ejemplo convincente de los roles a veces claves de personalidades: una persona de negocios emprendedora (Skinner) y un vicerrector emprendedor (Wilson). Es poco probable que el modelo CQU-CMS se replique, pero sirve como un ejemplo específico de una asociación público / privado abriendo nuevos caminos como las universidades australianas buscaban hacer frente a las demandas emergentes del neoliberalismo. Rodan, P. (2016).
En el Reino Unido; donde en la época contemporánea se inicia el impulso de las APP's, se refiere el caso de estudio denominado "The re-development of the Cruciform Building”, the University College de Londres, este proyecto inició en 1992 debido a la necesidad de mejorar la infraestructura de la escuela de medicina que había sido ampliada al fusionarse la Escuela de Medicina de la UCL, la Real Escuela Libre de Medicina del Hospital [RFHSM] y los Institutos de postgrado de Salud Infantil, Neurología y Oftalmología, pero también por el deseo de contar con un espacio adicional para la investigación, este proyecto no fue concebido desde un principio como una PFI, que es el nombre usado en el Reino Unido para estas asociaciones público privadas.

Es importante resaltar a continuación algunos de las lecciones que se generaron a partir de esta asociación destacándose entre ellas: "las negociaciones se retrasaron con frecuencia después de que el prestamista se enganchó porque los puntos previamente asentados entre la universidad y el socio del sector privado eran inaceptables para los grupos de interés más adelante. Esto podría haberse evitado si el prestamista o patrocinador participa activamente desde el inicio de la alianza. Este es un problema común en PFI. («PFI Case Study The ReDevelopment of the Cruciform Building. University College London», s. f.) 
renovación del campus, así como reducir el costo y mejorar la calidad de los servicios de comedor ofreció, Gilroy, L., Kenny, H., Snell, L., Ybarra, S., \& Millhouse, T. (20II), para el año 2014 en California “ la Universidad de California-Riverside emitió una solicitud de propuestas que buscan un socio privado para hacerse cargo de las operaciones de la campus de la librería de julio de 2014", la figura de APP ha sido utilizada no solo para beneficios de las Instituciones sino también para crear nuevos espacios para la comunidad local como tal y como ocurrió en Florida "En agosto de 2013, la Junta de Distrito del Colegio Seminole Estado de Síndicos anunciado planes para dos APP.

La primera implicaría una expansión multifase de su campus Altamonte Springs para implementar un plan maestro para desarrollar nueve nuevos edificios en el campus de la adición de I,4 millones de pies cuadrados de espacio, un segundo proyecto se desarrollaría un nuevo centro de salud en el campus de Sanford / Lake Mary para servir a los estudiantes, empleados y la comunidad circundante. Ese mismo mes, la Universidad de West Florida seleccionado Balfour Beatty Soluciones de campus como el desarrollador principal de 10 años, $\$ 500$ millones para expansión de sus principales planes del campus que implica para una nueva asociación de estudiantes, alojamiento para alumnos, vivienda activo adulto, estadio de fútbol, garaje, plaza y campanario.”. (Leonard Gilroy y Lisa Snell 2014).

\section{b. Centros Cooperativos de} Investigación (CRC) : En el año 2004 la OECD destacó la política de innovación que se estaba presentando en Australia donde juegan un papel importante las Asociaciones Publicas Privadas y en especial la que se venían desarrollando para el funcionamiento de los CRC, los cuales nacieron en 1990 como un programa para impulsar la investigación, cada CRC es una empresa de colaboración a largo plazo que involucra organizaciones que realizan investigaciones, como universidades 0 institutos públicos de investigación, y usuarios de investigación, como la industria. En algunos de los "públicos" buenos "CRC, el usuario de la investigación es la organización relevante del sector público o el departamento gubernamental y no incluye el sector privado. Por lo tanto, aunque la mayoría de los CRC son verdaderas asociaciones público / privadas, algunos son asociaciones entre el investigador público y el usuario público de investigación. OECD, O. (2004).

En cuanto a la Educación y el entrenamiento, "el CRC propuesto tiene un programa bien desarrollado de educación y capacitación de postgrado orientado a usuarios de investigación y las necesidades de la industria. El programa de educación y capacitación mejorará de manera demostrable las perspectivas de empleo y el valor de los graduados del programa en la industria y el entorno del usuario". OECD, O. (2004).
La Asociaciones Públicos privadas en el Reino Unido han venido siendo últimamente criticadas puesto que el sector privado ha intentado transferir el riego al sector público, siendo importante indicar que "Las APP del Reino Unido son financiadas inicialmente por bancos y capital privado, que exigen una 'prima de riesgo' durante la construcción fase para compensar las pérdidas potenciales, por ejemplo, riesgo de que una empresa de construcción vaya a la quiebra y el activo no será construido. Una vez que se completa la construcción y el riesgo de construcción se evapora, el consorcio PPP refinancia el proyecto, a tasas de interés más bajas, con acciones de propiedad que se transfieren a fondos de pensiones y inversores institucionales a largo plazo, que requieren un bajo riesgo estable devoluciones" ("The UK's PPPs Disaster: Lessons on Private Finance for the World» 2017). 
El proyecto de la Universidad Privada (Universidad Católica de América) y la entidad Inmobiliaria (JLL) dicha institución se encuentra en el centro de Washington D.C, cuyo objetivo inicial era re urbanizar 8 de los 176 acres que poseía, mejorando así su campus y apoyando las artes principalmente. Los principales resultados de la APP se dieron "Con el enfoque reflexivo para abordar un conjunto tan grande de objetivos, y la asociación público-privada correcta, la Universidad Católica ha encontrado capaz de desbloquear el valor máximo de su propiedad mientras cumplir sus objetivos estratégicos, principalmente: crear un ingreso stream, un búfer de seguridad alrededor de la escuela, y una entrada más atractiva al campus, el proyecto de la Universidad Católica, apodado "Monroe Street Market" es un lugar vibrante, área conectada de uso mixto que incluye 720 unidades residenciales, 45 casas adosadas, 83,000 pies cuadrados de tiendas en el nivel de la calle, 15,000 pies cuadrados de espacio de estudio artístico, un estudio de danza comunitaria de 3,000 pies cuadrados, y amplio aparcamiento. Los planes mejoran la transitabilidad y los patrones de tráfico." Lang. J, Inc (20I6).
Este tipo de Asociaciones público privadas se siguen impulsando en el año 2018 por la oficina de Economía del gobierno de Australia a través del Departamento de Industria, Innovación y Ciencia. "Los CRC tiene dos programas; el primero las colaboraciones dirigidas por la industria a mediano y largo plazo por hasta 10 años. No hay un límite establecido para la financiación de CRC; la segunda la que llevan a cabo investigaciones colaborativas a corto plazo, dirigidas por la industria, por hasta tres años. Las subvenciones de CRC-P están limitadas a un máximo de $\$$ 3 millones. Fueron presentados en 2015." (Australian Goverment, Departament of industry, innovation and science, s. f.)
El Reino Unido ha hecho dos intentos para introducir nuevos modelos de infraestructura para reemplazar PFI después de su crítica generalizada. La distribución sin fines de lucro (NPD), desde 2007 en Escocia, y PF2 en Inglaterra y Gales a partir de 2012. Sin embargo, ninguno de los modelos trata adecuadamente los defectos fundamentales de las PPP, es decir, costosas finanzas privadas, y costosas, inflexibles prestaciones de servicios privados. («The UK's PPPs Disaster: Lessons on Private Finance for the World» 2017).

Fuente: Investigador

La tabla 03, refiere a los casos desde las 3 grandes regiones Estados Unidos, Australia y el reino Unido, en cada uno de estas referencias se encuentran argumentos claves para la toma de decisiones de los involucrados, se involucra el aspecto de relación de las alianzas público privadas con las universidades, se rescata el papel de las oficinas de transferencia tecnológica de las universidades, cada región establece dinámicas y temas diversos de aplicación en el sector productivo, económico, educativo.

\section{Asociaciones público- privadas en Colombia para educación superior caso de análisis}

En Colombia las asociaciones público privadas fueron reguladas mediante la ley 1508 de 2012 y sus decretos reglamentarios, sin que esto quiera decir que con anterioridad a esta normatividad no se realizaban Alianzas o Asociaciones entre las entidades públicas y las privadas, No obstante, lo anterior, en materia de educación superior las APP no ha tenido un mayor desarrollo, el intento que hasta la fecha se ha presentado de Asociación Publico Privadas para la Educación Superior teniendo con fundamento en la ley 1508 de 2012 y, posteriormente las Asociaciones y Alianzas que se han venido desarrollando basadas en el art. 355 de la Constitución Política en cuanto a educación superior se refiriere. APP basadas en la ley 1508 de 20I2, en Colombia en el año 2012 la ley 1508 estableció el régimen de Asociaciones público privadas, dicha ley como anteriormente se ha indicado, ha venido siendo reglamentada por diversos decretos y se han realizado por el Departamento Nacional de Planeación (DNP).

El primer intento en desarrollar una Asociación Publico Privada en Educación Superior, cuyo objetivo era "El Diseño,
Construcción, Operación y Mantenimiento de una nueva infraestructura educativa, el centro de servicios universitarios en el Sur de Bogotá -CSU", dicha APP se presenta como de iniciativa privada sin recursos públicos, siendo liderada por la Corporación Universitaria Minuto de Dios en conjunto con mercantil Colpatria y donde el aporte solicitado a la Alcaldía Mayor de Bogotá era un lote en localidad de Ciudad Bolívar que había sido entregado a la Secretaria de Educación de Distrital, aspecto importante que el aporte en terrenos da el primer paso en la consecución de estas APP.

Este proyecto de APP surge en el año 2009 a través de una propuesta de UNIMINUTO para contribuir con el desarrollo integral y la disminución de pobreza de las familias que habitan el sur de la ciudad de Bogotá a través del acceso a la formación en Educación Superior que les permita mejorar su desarrollo personal, su nivel de ingreso y su calidad de vida y para esto se trazan dos objetivos específicos; el primero ofrecer formación técnica y tecnológica a 10.000 jóvenes del Sur de Bogotá a través del establecimiento de la 
Sede UNIMINUTO Bogotá Sur y segundo implementando un centro de servicios tecnológicos en el Sur de Bogotá que incluía Biblioteca, Laboratorios Especiales, Tecnología, Área de Bienestar, Servicios Administrativos de tal forma que el estudiante pudiera encontrar centralizados los servicios complementarios a las clases para su mayor comodidad, y para facilitar su asistencia diaria a las aulas, se articularía con 10 colegios de las localidades de Tunjuelito, Ciudad Bolívar, Bosa, Puente Aranda y Usme, donde ya UNIMINUTO hacia presencia en algunos de ellos para dicho momento gracias a una alianza con la Secretaria de Educación Distrital.

En el año 2010 UNIMINUTO y MERCANTIL COLPATRIA realizan una alianza estratégica, cuyo objeto es unir esfuerzos para lograr el desarrollo y consolidación de la sede Bogotá Sur de UNIMINUTO, comenzando en ese año a realizarse una serie de donaciones con las que se constituye un Fondo Fiduciario para recibir futuros aportes al proyecto, es importante reconocer como esta unidad administrativa y financiera garantizaría las rutas de sostenibilidad del proyecto, así mismo, se formaliza la participación en el Consejo Superior de UNIMINUTO con dos miembros designados por MERCANTIL COLPATRIA quien a su vez propone construir la sede en terrenos que debía entregar el proyecto Madelena de Constructora COLPATRIA al Distrito Capital destinados para equipamiento urbano, donde se aprovecha el terreno ofrecido por las alianzas.

Según (Gustavo Tamayo A., Pedro Valencia, y Andrés Hidalgo 20I2)., el análisis normativo; (i) Tanto la Ley 1508 de 2012 como el Decreto Reglamentario establecen un procedimiento claro y con tiempos definidos para la presentación de la iniciativa privada, que permiten a Colpatria/ UNIMINUTO estructurar el Proyecto de una manera más clara y con mayor certeza respecto de los procedimientos aplicables. (ii) El aporte del inmueble por parte del Distrito se encuentra expresamente previsto en la legislación aplicable sin que se considere una erogación de recursos públicos en los términos del Decreto Reglamentario, sin perjuicio de la obligación de revertir tanto el inmueble como la infraestructura

\section{Discusión}

Las Alianzas o Asociaciones Público- Privadas APP, Al-Said Albella, A. (20I2), son consideradas bienes servicios y alianzas transformacionales, que enfatizan en argumentar objetivos sociales de gran impacto, la establece como la confluencia de actores estatales, privados, sociedad civil, que tiene como propósito una agenda compartida de interés común, las APP se reconocen en la teoría de la modernización por el valor en la integración social, desde la economía a los sectores públicos y privados, logrando sociedades modernas más productivas, se articula en el trabajo y aplicación del capital social como resultado de la gestión, aplicados como el factor diferencial de estos acuerdos, debido a que desde la teoría el capital social parte del capital cultural inherente en la sociedad, su relación con la escuela y como este genera un espacio social determinado para el desarrollo, las alianzas y acuerdos por el desarrollo no son totalmente independiente del capital económico y cultural de un individuo determinado, desde la autodeterminación como estrategia de consolidación construida y la dotación correspondiente al Proyecto al finalizar el Contrato de APP. (iii) Siempre y cuando no existan aportes de naturaleza pública al Proyecto (toda vez que el aporte del inmueble se encuentra expresamente excluido como tal bajo el Decreto Reglamentario), no se generaría la obligación de acudir a un proceso licitatorio sino a un proceso abreviado de contratación en el peor de los casos."

En el mes de noviembre del año 20I2, se radicó el Estudio de Pre factibilidad de Alianza Público Privada - APP, presentándose como una Asociación Público Privada de iniciativa privada sin recursos públicos, en donde el centro de servicios universitarios estaría diferenciado por el grado de innovación en la oferta de programas y los servicios de desarrollo a las comunidades, contemplando la actividad de educación superior, junto con el proyecto grado 12 y el de Bilingüismo que coincidían con los lineamientos del Plan de Desarrollo de la ciudad en ese momento. Esta propuesta iba dirigida a una población entre los 16 y 25 años y los Estratos 1 y 2 de Sur de Bogotá, en donde la ejecución del proyecto se retribuiría con el derecho para que COLPATRIA/ UNIMINUTO explotará tal infraestructura o servicio durante aproximadamente 30 años acorde a lo que indica la norma, la APP se le realiza un primer requerimiento de aclaraciones por la Secretaria de Educación en el mes de enero de 2013 y es hasta el mes de mayo de dicho año en donde se hace ya una revisión a la etapa de pre factibilidad emitiéndose un concepto por cada una de las direcciones que conforman esa Secretaría, al cual se le da respuesta por parte de las entidades que presentaron la iniciativa. Cabe resaltar que en Colombia a la fecha no se han presentado nuevos intentos de asociaciones públicos privadas en materia de Educación superior, no obstante en cuanto a Educación básica y secundaria se refiere se encuentra en etapa de factibilidad los primeros colegios públicos que se están llevando a cabo por la iniciativa pública del Ministerio de Educación Nacional, quien ha visto en estas Asociaciones una gran posibilidad para la Construcción, dotación, operación, mantenimiento y prestación de servicios no pedagógicos de colegios en diversos municipios del país.

y consecución de estas estrategias. Constituye un aporte significativo al entendimiento del éxito o el fracaso de los acuerdos en los sectores públicos y privados, posibilitando la elección estratégica como el aporte desde el conocimiento integral de la organización, que mediante alianzas, acuerdos y espacios voluntarios integran el conocimiento a su sector más práctico y especifico, no obstante, se refiere las APP, un aspecto de la Visión directiva y elección estratégica.

El funcionamiento y aplicación de las figuras jurídicas varían de acuerdo con el marco legal del país, sus resultados obedecen a las voluntades políticas del desarrollo, la administración y elección tiene variables no controladas en su administración, se presenta que en el mundo se da un aporte donde estas políticas permiten que las universidades deben establecer y participar en la solución de los problemas irresolubles, aplicando las ciencias fácticas en la construcción de caminos de desarrollo. 


\section{Conclusiones}

Para lograr una alianza publico privada se requiere de un escenario jurídico administrativo favorable a las condiciones, determinado por la normatividad de acuerdo a la zona geográfica, localidad, se establece un dialogo de actores que estén de acuerdo con el lineamiento, la política existente, luego de su puesta en marcha se debe ampliar el valor de la determinación de estos proyectos, el desarrollo y la respuesta a problemáticas comunes, Colombia comparte con Chile los entornos más favorables para el desarrollo de una infraestructura de APP, desde las modalidades jurídicas mencionadas los convenios, el Jointventure, fcilitando el desarrollo, esto se relaciona en el EIU, E. I. U. (2017), The 2017 Infrascope: Evaluating the Environment for PublicPrivate Partnerships in Latin America and the Caribbean, no obstante lo demostrado en la investigación reconoce que no existen casos de éxito, y este fenómeno se incrementa en las universidades colombianas.

Las alianzas público privadas (APP) logran la aplicación de las teorías de la inclusión social y del capital social de Pierre Bourdieu, cuando establecen y priorizan problemáticas del común que favorecen el sector productivo de una región, logran la aplicación de la autodeterminación cuando sus resultados están ligados a un bienestar mutuo, establecen un dialogo directivo visionario cuando realizan la elección estratégica como un marco integrador para entender este fenómeno ratificados en casos como el de la cooperación realizada entre Coca-Cola HBC Serbia ad Zemun y la Facultad de Ciencias de la organización (FOS), en la Universidad de Belgrado, las empresas multinacionales invierten en educación, el argumento más importante la cooperación que indirectamente mejora la reputación académica del claustro universitario si no que aporta a la responsabilidad social empresarial de ambas partes, este posicionamiento de las facultades promueve el desarrollo de APP, es allí donde se resalta el papel de las universidades, el estados, las empresas.

Las asociaciones público privadas en educación superior en Colombia deben tener en cuenta los ejemplos y las lecciones aprendidas que en esta materia se han presentado tanto a nivel nacional como internacional, tomando lo mejor de estas sin desconocer la realidad local del territorio donde se lleven a cabo las mismas, se debe estructurar figuras jurídicas acordes con el propósito, facilitando la estructuralidad y consistencia que logre garantizar su implementación y desarrollo, fortaleciendo la práctica educativa como se logra que se genere una Universidad multidisciplinaria como una institución educativa de apoyo regional en términos de asociación público-privada según Dugarova, D. T., Kimova, S. Z., \& Dambayeva, B. B. (2015), la consolidación del sistema de educación superior, y la creación de una infraestructura moderna para la interconexión de la actividades científicas, educativas y productivas, posibilitando la transferencia tecnológica.

Las oficinas de transferencia tecnológica, científica e investigativa de las instituciones de educación superior, se convierten en las unidades estratégicas de búsqueda de alianzas con el sector productivo, se fomenta la educación dual, la priorización de soluciones de problemas irresolubles pertinentes para su misionalidad, las APP facilitan la interacción con los aspectos de desarrollo y visibilidad de la educación y su papel en el desarrollo latinoamericano. 


\section{Referencias bibliográficas}

Adams, A. M., Rabbani, A., Ahmed, S., Mahmood, S. S., Al-Sabir, A., Rashid, S. F., \& Evans, T. G. (2013). Explaining equity gains in child survival in Bangladesh: scale, speed, and selectivity in health and development. The Lancet, 382(9909), $2027-2037$.

Aguja, F. A. P., \& Quintero, H. J. G. (20I7). Modelo de gestión del conocimiento solidario, LA RUTA HACIA LA INNOVACIÓN SOCIAL. REVISTA CIENTÍFICA AXIOMA, (16), 49-59.

Al-Said Albella, A. (20I2). Las alianzas público-privadas para el desarrollo: aproximación al marco teórico.

Bourdieu, P. (1997). Capital cultural, escuela y espacio social. Siglo xxi.

CEPAL, N. (20I7). La Inversión Extranjera Directa en América Latina y el Caribe 2017.

Davis, N. Z., \& Luna, P. M. (20I3). Descentralizando la historia: relatos locales y cruces culturales en un mundo globalizado. Historia Social, 165-179.

Dugarova, D. T., Kimova, S. Z., \& Dambayeva, B. B. (20I5). On Educational Practice Dissemination of Multidisciplinary University as a Regional Support Higher Educational Institution in Terms of Public-Private Partnership. Indian Journal of Science and Technology, 8(s (I0)).

Erasso Cifuentes, M. C., Pérez Soto, M., Pimienta Tabera, J. G., Rendón Ossa, E., \& Vásquez Zapata, C. D. (20I4). Conocimiento de las administraciones de los municipios del Valle de Aburrá sobre las asociaciones público privadas (Bachelor's thesis, Universidad de Medellín).

Erazo Chapid, Edison. 2017. «Las Asociaciones Publico Privadas Institucionales En Colombia». instname:Universidad del Rosario, febrero. http://repository.urosario.edu.co/handle/I0336/I 32 I 3.

Erazo Chapid, E. (2017). Las Asociaciones Publico Privadas Institucionales En Colombia.

Erazo Chapid, Edison. 2017. «Las Asociaciones Publico Privadas Institucionales En Colombia». instname:Universidad del Rosario, febrero. http://repository.urosario.edu.co/handle/I0336/I3213.

Estrada, V. (20I7). ESTUDIO COMPARATIVO EN LA IMPLEMENTACIÓN DE LAS ASOCIACIONES PÚBLICO-PRIVADAS (APP).

EIU, E. I. U. (2017). The 2017 Infrascope: Evaluating the Environment for Public-Private Partnerships in Latin America and the Caribbean.

Gilroy, L., Kenny, H., Snell, L., Ybarra, S., \& Millhouse, T. (20I I). Annual Privatization Report 20I0: State Government Privatization. Reason Foundation. http://reason. org/files/state_annual_privatization_report_2010.pdf.

Growe, D. (2010). United Nations Foundation. In International Encyclopedia of Civil Society (pp. I594- I595). Springer, New York, NY.

Growe, D. (2010). United Nations Foundation. In International Encyclopedia of Civil Society (pp. I594- I595). Springer, New York, NY

Hernández Sampieri, R., Fernández Collado, C., \& Baptista Lucio, P. (2010). Metodología de la investigación.

Horner, S., Jayawarna, D., Giordano, B., \& Jones, O. (2019). Strategic choice in universities: Managerial agency and effective technology transfer. Research Policy.

Iglesias, J. L. V. (2008). Comentarios a la Ley 30/2007, de 30 de octubre, de contratos del sector público. La Ley.

Legislativo, O. (2012). Asociaciones Público Privadas Ley 1508 de 2012. Boletín, 197.

Paletta, A., Fava, F., Ubertini, F., Bastioli, C., Gregori, G., La Camera, F., \& Douvan, A. R. (20I9). Universities, industries and sustainable development: Outcomes of the 2017 G7 Environment Ministerial Meeting. Sustainable Production and Consumption.

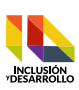


OECD, O. (2004). The OECD principles of corporate governance. Contaduría y Administración, (2 I6).

Robert H. Edwards Jr., Mark J. Riedy, Kilpatrick Townsend. apr20I5. «The Public-Private Partnership Law Review - United States | Public Private Partnership». apr2015. http://ppp.worldbank.org/public-private-partnership/library/public-private-partnership-lawreview-united-states.

Rodan, P. (20 I6). Public-private partnership in higher education: Central Queensland University meets Campus Management Services. Australian Universities' Review, The, 58(I), 5.

Ryan, R., \& Deci, E. L. (2000). La Teoría de la Autodeterminación y la Facilitación de la Motivación Intrínseca, el Desarrollo Social, y el Bienestar. American Psychologist, 55(I), 68-78.

Sepúlveda Figueroa, B. J. (20I4). Las asociaciones público privadas y el contrato de concesión: Regulación parcial en la Ley I508 de 2012 (Doctoral dissertation, Universidad Nacional de Colombia).

Sepúlveda, B., \& BLANCA, J. (20I4). Las asociaciones público privadas y el contrato de concesión: Regulación parcial en la Ley I508 de 20I2. Trabajo de grado.

Solheim-Kile, E., \& Wald, A. (2019). Extending the Transactional View on Public-Private Partnership Projects: Role of Relational and Motivational Aspects in Goal Alignment. Journal of Construction Engineering and Management, I45(5), 040 I 9030.

Urueta Rojas, Juan Manuel. 2010. El contrato de concesión de obras públicas. Biblioteca Juridica Otto Morales Benitez. Bogotá: Grupo Editorial lbañez.

Vandepoorter, A. (20I4). Concession d'aménagement ou contrat de partenariat. Contrats Publics, (I45), 60-64.

Vásquez Jaramillo, F. (20I7). Fallas y retos del modelo de Asociaciones Público-Privadas para el desarrollo de la infraestructura social (Bachelor's thesis, Universidad EAFIT)

Zapata Rotundo, G. J., Canet Giner, M. T., \& Peris Bonet, F. J. (2008). Visión directiva y elección estratégica: su influencia en los tipos básicos de trabajo. Cuadernos de Administración, 2 I (37).

Zapata Rotundo, G. J., Mirabal, A., \& Canet Giner, M. T. (20I5). El entorno de la organización: un estudio de sus tipologías y su vinculación con la percepción directiva y el diseño organizativo. Ciencia y Sociedad, 40(4). 